\title{
Aligned multi-walled carbon nanotubes with nanohydroxyapatite in a 3D printed polycaprolactone scaffold stimulates osteogenic differentiation
} DOI:

10.1016/j.msec.2019.110374

\section{Document Version}

Final published version

Link to publication record in Manchester Research Explorer

Citation for published version (APA):

Huang, B., Vyas, C., Byun, J. J., El-Newehy, M., Huang, Z., \& Da Silva Bartolo, P. J. (2020). Aligned multi-walled carbon nanotubes with nanohydroxyapatite in a $3 \mathrm{D}$ printed polycaprolactone scaffold stimulates osteogenic differentiation. Materials Science and Engineering C, 108, [110374]. https://doi.org/10.1016/j.msec.2019.110374

\section{Published in:}

Materials Science and Engineering C

\section{Citing this paper}

Please note that where the full-text provided on Manchester Research Explorer is the Author Accepted Manuscript or Proof version this may differ from the final Published version. If citing, it is advised that you check and use the publisher's definitive version.

\section{General rights}

Copyright and moral rights for the publications made accessible in the Research Explorer are retained by the authors and/or other copyright owners and it is a condition of accessing publications that users recognise and abide by the legal requirements associated with these rights.

\section{Takedown policy}

If you believe that this document breaches copyright please refer to the University of Manchester's Takedown Procedures [http://man.ac.uk/04Y6Bo] or contact uml.scholarlycommunications@manchester.ac.uk providing relevant details, so we can investigate your claim.

\section{OPEN ACCESS}




\title{
Aligned multi-walled carbon nanotubes with nanohydroxyapatite in a 3D printed polycaprolactone scaffold stimulates osteogenic differentiation
}

\author{
Paulo Bártolo ${ }^{\mathrm{a}, *}$ \\ a School of Mechanical, Aerospace and Civil Engineering, M13 9PL, University of Manchester, Manchester, UK \\ ${ }^{\mathrm{b}}$ School of Materials, The University of Manchester, Manchester, M13 9PL, UK \\ ${ }^{\mathrm{c}}$ Department of Chemistry, College of Science, King Saud University, Riyad, 11451, Saudi Arabia \\ ${ }^{\mathrm{d}}$ Department of Mineral Engineering, Central South University, Changsha, 410083, PR China
}

Boyang Huang ${ }^{\mathrm{a}}$, Cian Vyas ${ }^{\mathrm{a}}$, Jae Jong Byun ${ }^{\mathrm{b}}$, Mohamed El-Newehy ${ }^{\mathrm{c}}$, Zhucheng Huang ${ }^{\mathrm{d}, * *}$,

\section{A R T I C L E I N F O}

\section{Keywords:}

Additive manufacturing

Bone scaffolds

Hierarchical structures

Hydroxyapatite

Multi-walled carbon nanotubes

\begin{abstract}
A B S T R A C T
The development of highly biomimetic scaffolds in terms of composition and structures, to repair or replace damaged bone tissues, is particularly relevant for tissue engineering. This paper investigates a 3D printed porous scaffold containing aligned multi-walled carbon nanotubes (MWCNTs) and nano-hydroxyapatite (nHA), mimicking the natural bone tissue from the nanoscale to macroscale level. MWCNTs with similar dimensions as collagen fibres are coupled with nHA and mixed within a polycaprolactone (PCL) matrix to produce scaffolds using a screw-assisted extrusion-based additive manufacturing system. Scaffolds with different material compositions were extensively characterised from morphological, mechanical and biological points of views. Transmission electron microscopy and polarised Raman spectroscopy confirm the presence of aligned MWCNTs within the printed filaments. The PCL/HA/MWCNTs scaffold are similar to the nanostructure of native bone and shows overall increased mechanical properties, cell proliferation, osteogenic differentiation and scaffold mineralisation, indicating a promising approach for bone tissue regeneration.
\end{abstract}

\section{Introduction}

The main goal of tissue engineering is to repair, replace and restore damaged or diseased tissues through the use of biocompatible and biodegradable synthetic or natural materials, cells and biomolecular signals [1]. The selection of the right type of materials is a key issue as the aim is to create constructs mimicking the native extracellular matrix (ECM) of tissues [2-4]. In the case of bone, the ECM consists of organic collagen and inorganic bone mineral hierarchically organised across multiple length scales [5,6]. Various biomaterials have been explored as synthetic bone scaffolds. However, most strategies merely focus on the use of biomaterials mimicking the chemical composition of bone and considering its structural performance. However, challenges still remain mainly related to complex fabrication processes [7], lack of reproducibility [8], unmanaged pore characteristics [9] and poor mechanical properties [10]. Therefore, the design and fabrication of biocompatible and biodegradable bone scaffolds mimicking the hierarchical structure of native bone remains a critical challenge.
Hydroxyapatite (HA) is a bioceramic material, widely used for clinical bone grafting due to its similar chemical composition to native apatite in the human skeleton. It presents excellent biocompatibility, bioactivity and osteoconductivity [11]. Carbon nanotubes (CNT) have extraordinary mechanical and electrical properties and are particularly relevant for bone tissue engineering applications due to their unique dimensional characteristics similar to the fibrillar proteins (e.g. collagen) present in bone. They can be fabricated in a wide range of diameters and lengths comparable to tropocollagen, $1.5 \mathrm{~nm}$ in diameter and $300 \mathrm{~nm}$ in length, and collagen fibrils and fibres, $20-400 \mathrm{~nm}$ in diameter and lengths up to hundreds of microns $[5,12,13]$.

The combined use of CNTs and HA has been reported. Liao et al. [14] investigated a self-assembled nano-HA (nHA) on multi-walled carbon nanotubes (MWCNTs), showing that the multi-walled surfaces of the CNTs provide abundant nucleation sites for apatite formation. Oyefusi et al. [15] grafted HA on carboxylated CNTs and conducted cell proliferation and differentiation studies using human fetal osteoblasts. No toxic effects were observed. Yoon et al. [16] mimicked collagen

\footnotetext{
** Corresponding author. School of Mechanical, Aerospace and Civil Engineering, Pariser Building, Room C4, The University of Manchester, Sackville Street, M13 BB, Manchester, UK.

${ }^{* *}$ Corresponding author. Department of Mineral Engineering, Peace Building. Room 250, Central South University, 410083, Changsha, PR China.

E-mail addresses: zchuang@csu.edu.cn (Z. Huang), paulojorge.dasilvabartolo@manchester.ac.uk (P. Bártolo).
} 
fibrils by covalently grafting gelatin onto functionalised CNTs followed by the assembly of HA onto the gelatin-CNTs using a simulated body fluid solution. Results showed that the tensile strength, elastic modulus, and elongation rate of the composite material were significantly improved compared to pure gelatin-CNTs and cell viability studies using rat mesenchymal stem cells showed improved biocompatibility. Although the combined use of CNTs with HA shows great potential for bone tissue engineering applications, some limitations remain unsolved. In the case of non-covalent binding, the relatively weak bonds lead to easily detachment of the ceramics from the CNTs [14]. Moreover, the covalent binding of HA to the CNT walls might change the surface and mechanical characteristics of CNTs [17]. Besides, these materials are difficult to process and consequently reported studies focus on two-dimensional (2D) membranes, which cannot mimic the complexity of the three-dimensional (3D) bone environment. Recently, some researchers reported the use of extrusion printing, freeze casting and soft method to produce 3D HA scaffolds containing CNTs or graphene. However, these are laboratory techniques that not allow controlling the topology of the produced scaffolds, pore shape and porosity and the distribution of CNTs and graphene in the scaffolds [18-20].

This paper investigates the use of a screw-assisted extrusion-based additive manufacturing system to produce hierarchical scaffolds using biomaterials able to mimic both bone composition and bone structure from nano to macro level. In the produced 3D porous hierarchical scaffolds, MWCNTs mimic the highly aligned fibril collagens and synthetic nHAs are similar to the native HAs within a polycaprolactone (PCL) matrix. The effects of adding MWCNTs and nHAs on morphological, physical and biological properties are also extensively investigated.

\section{Methods}

\subsection{Materials}

MWCNTs were fabricated with a diameter of $20 \mathrm{~nm}$ and average length of $10 \mu \mathrm{m}$ via a catalytic vapour deposition (CVD) method, as previously reported [21]. PCL was supplied by PerstorpCaprolactones (UK). nHA, phosphate buffered saline (PBS), glutaraldehyde, hexamethyldisilazane (HMDS) and 10\% neutral buffered formalin, Triton X100, Alizarin red-S (ARS), $10 \%$ ammonium hydroxide, radioimmunoprecipitation assay (RIPA) buffer, and protease inhibitor were all purchased from Sigma-Aldrich (UK). Human adipose-derived stem cells (hADSCs), MesenPRO RS ${ }^{\mathrm{TM}}$ basal media, STEMPRO ${ }^{\mathrm{TM}}$ osteogenesis differentiation kit, Alexa Fluor 488-conjugated phalloidin, 4',6-diamidino-2-phenylindole (DAPI), and the bicinchoninic acid assay (BCA, Micro BCA Protein Assay Kit) were all obtained from Thermo Fisher Scientific (UK). SensoLyte ${ }^{\circledR}$ pNPP Alkaline Phosphatase (ALP) assay kit was purchased from AnaSpec (USA). Osteocalcin (OCN) Human SimpleStep ELISA ${ }^{\circledast}$ Kit was obtained from Abcam (UK). Sirus Red/Fast Green Collagen staining kit (COL) was purchased from Chrondrex (USA). Adipose derived stem cells were used instead of bone marrow mesenchymal stem cells, as they are more accessible and are able to differentiate into osteoblasts. Overall, these appear to be little difference between the potential of these cells and those derived from bone marrow cells. Moreover, the field of stem cells from adipose tissue can be greater than from bone marrow cells as a large volume of fat can be isolated.

\subsection{Scaffold fabrication}

Nanocomposite materials were prepared using a melt blending method and the following compositions were considered: PCL/MWCNT (99.25/0.75 wt \%), PCL/HA (80/20 wt \%), and PCL/HA/MWCNT (79.25/20/0.75 wt \%). PCL pellets were melted at $100{ }^{\circ} \mathrm{C}$ and the nHA and MWCNTs were added to the melted polymer and manually mixed for $30 \mathrm{~min}$. A screw-assisted extrusion additive manufacturing system
(3D Discovery, RegenHU, Villaz-Saint-Pierre, Switzerland) was used to produce the scaffolds using the following processing conditions: air pressure of $6 \mathrm{bar}$, melting temperature of $90^{\circ} \mathrm{C}$, deposition velocity of $20 \mathrm{~mm} / \mathrm{s}$, and screw rotational velocity of $12 \mathrm{rpm}$. Scaffolds were designed with a $0 / 90^{\circ}$ lay-down pattern, $330 \mu \mathrm{m}$ filament diameter, and $350 \mu \mathrm{m}$ pore size.

\subsection{Morphological analysis}

The morphology and composition of the scaffolds was investigated using scanning electron microscopy (SEM), Nova nanoSEM (FEI, Hillsboro, USA), coupled with energy dispersive spectroscopy (EDS). Atomic force microscopy (AFM), MultiModeV (Bruker, Massachusetts, USA), was used to investigate the topographic characteristics of the scaffold filaments $(n \geq 5)$. The surface roughness is presented as the arithmetic mean value Ra, considering at least five measurements for each scaffold.

\subsection{Transmission electron microscopy (TEM)}

TEM (HT7700, Hitachi, Japan) was used to investigate the internal structure of the nHA and MWCNTs in the PCL matrix. Ultra-thin samples were prepared by ultra-microtome, Leica EM UC7 (Leica Microsystems, Wetzlar, Germany), cut along the printing direction from the surface to the centre of the filament.

\subsection{Polarised Raman analysis}

Polarised Raman spectroscopy, HR Evolution Raman (LabRAM, Tokyo, Japan), was used to determine the alignment of MWCNTs in the printed filament as well as pre-processed composite blends. The second order $\mathrm{G}^{\prime}$ peak intensity $\left(\sim 2700 \mathrm{~cm}^{-1}\right)$ was used as an indication of the alignment of MWCNTs [22,23]. A $2 \mathrm{~mW}$ helium neon laser with a wavelength of $633 \mathrm{~nm}$, coupled with a phase filter (RSP05/M, THORLABS, UK), was used to obtain the Raman spectrum. A series of phase angles ranging from $0^{\circ}$ to $90^{\circ}$ with an interval of $22.5^{\circ}$ were selected using a polarised filter. The incident laser beam is parallel to the printing direction when the phase angle is $0^{\circ}$ and perpendicular to it when the phase angle is $90^{\circ}$. At least 15 random locations on both the printed filament and initial blends were scanned and the representative intensity at each phase angle was calculated by averaging the intensities obtained at each location. The schematic illustration is shown in Fig. 1. Polarised Raman mapping, showing the overall distribution of intensities of MWCNTs over a surface area $(120 \mu \mathrm{m} \times 180 \mu \mathrm{m})$ of a filament with an incident laser beam parallel to the printing direction (phase angle $=0^{\circ}$ ), was obtained by the accumulation of 400 discrete scans within that area. The semi-quantification of the intensity distribution was derived from the polarised Raman mapping and a histogram was produced using the software MATLAB (MathWorks, USA) by analysing the RGB colour pixels in accordance to the colour scale bar.

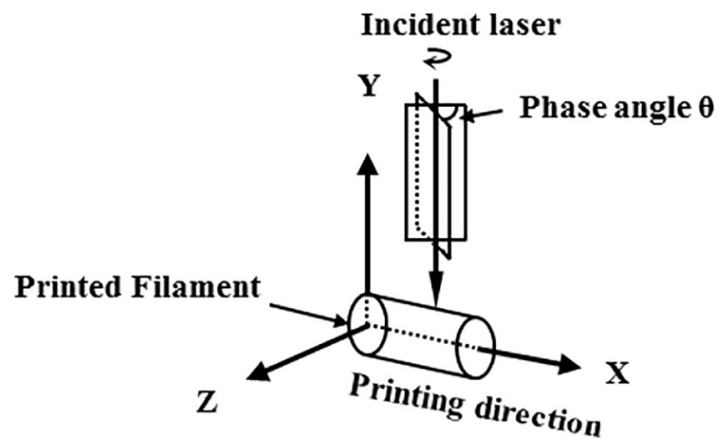

Fig. 1. Schematic illustration of the Polarised Raman analysis of printed filament. 
The level of MWCNTs orientation in each segment was semi-quantified by correlating the MWCNTs angle to the phase angle of the incident laser. Since Polarised Raman is not able to differentiate between + ve and -ve orientation differences, the MWCNTs orientation for each segment was classified as Segment A $=\left[-22.5^{\circ}, 22.5^{\circ}\right]$, Segment $\mathrm{B}=[-$ $\left.45^{\circ},-22.5^{\circ}\right] \cup\left[22.5^{\circ}, 45^{\circ}\right]$, Segment $C=\left[-67.5^{\circ},-45^{\circ}\right] \cup\left[45^{\circ}, 67.5^{\circ}\right]$, and Segment $\mathrm{D}=\left[-90^{\circ},-67.5^{\circ}\right] \cup\left[67.5^{\circ}, 90^{\circ}\right]$.

\subsection{Mechanical properties}

Compression tests were performed using the Instron 3344 (Instron, Massachusetts, USA). Scaffolds $(\mathrm{n}=9)$ were cut into $3 \mathrm{~mm} \times 3 \mathrm{~mm} \times 4 \mathrm{~mm}$ cubes and tested at a strain rate of $0.5 \mathrm{~mm} / \mathrm{min}$ with a $100 \mathrm{~N}$ load cell. The compressive modulus was obtained from the stress-strain curves and the yield strength was obtained by the intersected point of the parallel line to the elastic gradient with a strain offset of $0.2 \%$.

\subsection{In-vitro study}

Scaffolds were sterilised using $80 \%$ ethanol for $2 \mathrm{~h}$ and rinsed with PBS twice, followed by drying overnight. hADSCs, commonly used multipotent mesenchymal stem cells, were cultured by using MesenPRO $\mathrm{RS}^{\mathrm{TM}}$ basal media, $2 \%(\mathrm{v} / \mathrm{v})$ growth supplement, $1 \%(\mathrm{v} / \mathrm{v})$ glutamine, and $1 \%(\mathrm{v} / \mathrm{v})$ penicillin/streptomycin. The differentiation study used an osteogenesis differentiation basal medium supplemented with $10 \%$ (v/ v) osteogenic supplement and $1 \%(\mathrm{v} / \mathrm{v})$ penicillin/streptomycin. All samples in the differentiation study were cultured for 3 days prior to replacement of the cell culture media with differentiation media. Subsequently, all differentiation assays (ALP, COL, OCN, and ARS) use assay time-points of day $1,7,14$ and 28 which corresponds to a total culture time of day 4, 10, 17 and 31 . All scaffolds were seeded with $5 \times 10^{4}$ cells for cell proliferation and differentiation studies. Culture and osteogenic media was changed every 3 days.

\subsubsection{Cell proliferation}

Cell proliferation was assessed through the Alamar Blue assay, performed at days 1,3 and 7. At each time point, $50 \mu \mathrm{L}$ of $0.01 \%(\mathrm{v} / \mathrm{v})$ Alamar Blue solution was added to each sample $(n=4)$, incubated for $4 \mathrm{~h}$ and then the fluorescence measured using a microplate reader (540 $\mathrm{nm}$ excitation/590 $\mathrm{nm}$ emission wavelength).

\subsubsection{Alkaline phosphatase}

ALP activity, an indicator of early-stage osteogenesis, was measured at day 1, 7 and 14 using p-nitrophenyl phosphate (pNPP) substrate. The scaffolds $(n=4)$ were rinsed with PBS twice to remove the osteogenic media, followed by washing twice in $1 \mathrm{x}$ alkaline phosphatase dilution assay buffer. The scaffolds were lysed by $1 \mathrm{x}$ assay buffer with $0.2 \%$ (v/ v) Triton X-100. Samples were then vortexed for $30 \mathrm{~s}$, sonicated for $3 \mathrm{~min}$, and freeze-thawed twice at $-80^{\circ} \mathrm{C}$ for $20 \mathrm{~min}$. Samples were centrifuged at $2500 \times g$ for $10 \mathrm{~min}$ at $4{ }^{\circ} \mathrm{C} .50 \mu \mathrm{L}$ of the supernatant and $50 \mu \mathrm{L}$ of the pNPP substrate was mixed, gently shaken for $30 \mathrm{~s}$ and incubated for $1 \mathrm{~h}$. The absorbance was measured at $405 \mathrm{~nm}$ using a microplate reader. The concentration of ALP was calculated based on a standard curve and normalised to the total protein concentration which was determined by using the BCA.

\subsubsection{Collagen}

The amount of collagen (COL) was semi-quantified at day 14, 21 and 28, which was used as the mid-stage osteomarker. The scaffolds $(n=4)$ were rinsed with PBS and fixed by kahle fixative which was prepared by adding distilled water, $96 \%$ ethanol, 37\% formaldehyde and glacial acetic acid with a ratio of 30:14:5:1. After $15 \mathrm{~min}$ of incubation at room temperature, $0.2 \mathrm{~mL}$ of Dye Solution was added to stain the samples at room temperature for $30 \mathrm{~min}$. The stained samples were washed at least five times by distilled water, followed by adding the Dye Extraction Buffer on each sample and gently mixing by pipetting until the colour is eluted from the sample. $150 \mu \mathrm{L}$ of eluted dye solution was transferred to 96-well microplate and the absorbance was measured at $540 \mathrm{~nm}\left(\mathrm{OD}_{540}\right)$ and $605 \mathrm{~nm}\left(\mathrm{OD}_{605}\right)$ separately. The collagen concentration and non-collagenous protein concentration were calculated as follows:

$\mathrm{COL}(\mu \mathrm{g} / \mathrm{mL})=\left(\mathrm{OD}_{540}-\mathrm{OD}_{605} \times 0.291\right) / 0.0378$

Non-COL $(\mu \mathrm{g} / \mathrm{mL})=\mathrm{OD}_{605} / 0.00204$

The collagen concentration was normalised to the non-collagenous protein concentration.

\subsubsection{Osteocalcin}

The detection and quantification of OCN was performed at day 1, 7, 14 and 28. The scaffolds $(n=4)$ were washed with PBS and lysed in RIPA cell lysis buffer supplemented with protease inhibitor and shaken for $30 \mathrm{~min}\left(400 \mathrm{rpm}\right.$ and $\left.4{ }^{\circ} \mathrm{C}\right)$. The cell lysis solutions were centrifuged at $12,000 \times g$ at $4{ }^{\circ} \mathrm{C} .50 \mu \mathrm{L}$ of supernatant was added to a pre-coated 96 well microplate, followed by adding $50 \mu \mathrm{L}$ of an antibody cocktail. After incubation for $1 \mathrm{~h}$ at room temperature on a plate shaker at $400 \mathrm{rpm}$, the samples were gently washed 3 times by washing buffer and $100 \mu \mathrm{L}$ of TMB substrate solution was added and shaken in the dark for $10 \mathrm{~min}$. After adding $100 \mu \mathrm{L}$ of stop solution, the samples were read using a microplate reader at absorbance of $450 \mathrm{~nm}$. The concentration of OCN was calculated using a standard curve and normalised to the total protein concentration.

\subsubsection{Alizarin red staining}

Scaffold mineralisation was analysed by ARS at day 1, 7 and 14 . Two separate groups were tested with one group seeded with hADSCs and another group without cells. All samples were cultured with the same conditions. At each time point, samples $(n=4)$ were rinsed with PBS twice to remove the osteogenic media, followed by immersion in $10 \%$ formalin for $15 \mathrm{~min}$. The scaffolds were washed with deionised water three times and stained for $40 \mathrm{~min}$ with $0.2 \%$ (w/v) ARS staining solution. The residual ARS was removed by washing the scaffold five times with deionised water. $10 \%$ acetic acid solution was added to the samples for $30 \mathrm{~min}$ with gently shaking. The solution was removed from the scaffold, heated to $80^{\circ} \mathrm{C}$ for $10 \mathrm{~min}$, stored on ice for $15 \mathrm{~min}$, and eventually centrifuged for $5 \mathrm{~min}$ at $20,000 \times g$. $400 \mu \mathrm{L}$ of the supernatant was neutralised with $150 \mu \mathrm{L}$ of $10 \%$ ammonium hydroxide. The absorbance of the neutralised solution was detected by microplate reader at $405 \mathrm{~nm}$. The calcium mineralisation of only the cellular component of the seeded scaffold was obtained by subtracting the absorbance of the non-seeded samples from the total absorbance of scaffolds seeded with cells.

\subsubsection{Cell morphology}

The mineralisation process was assessed using both SEM and inverted fluorescence confocal microscopy (Leica DMI6000B, Leica Microsystems, Germany) at day 21. For SEM imaging, scaffolds were rinsed with PBS, fixed with $2.5 \%$ glutaraldehyde for $1 \mathrm{~h}$, and followed by a dehydration process using a series of ethanol concentrations from $50 \%$ to $100 \%$ (increments of $10 \%$ ) for $15 \mathrm{~min}$ each, with the $100 \%$ concentration repeated twice. Finally, scaffolds were immersed in a 50/ 50 mixture of ethanol and HMDS for 15 min and then $100 \%$ HMDS with samples allowed to dry overnight. EDS linear analysis was also performed to investigate potential surface composition changes along the scanning route. For confocal fluorescence imaging, scaffolds with cells were stained with phalloidin for actin staining and DAPI for nuclear staining. The samples were fixed with $10 \%$ formalin for $30 \mathrm{~min}$, washed twice with PBS, and followed by adding $0.1 \%$ Triton X-100 for 3 min to permeabilise the cell membrane. After washing with PBS three times, $8 \%$ fetal bovine serum (FBS) was added to block non-specific binding for 60 min. Phalloidin (1:400 dilution) with 1\% FBS solution was added 

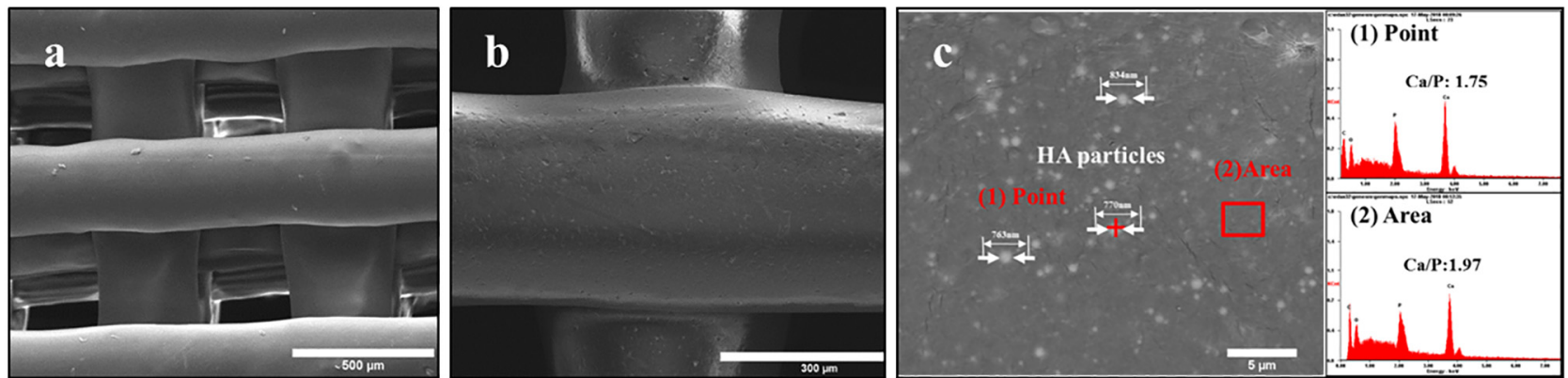
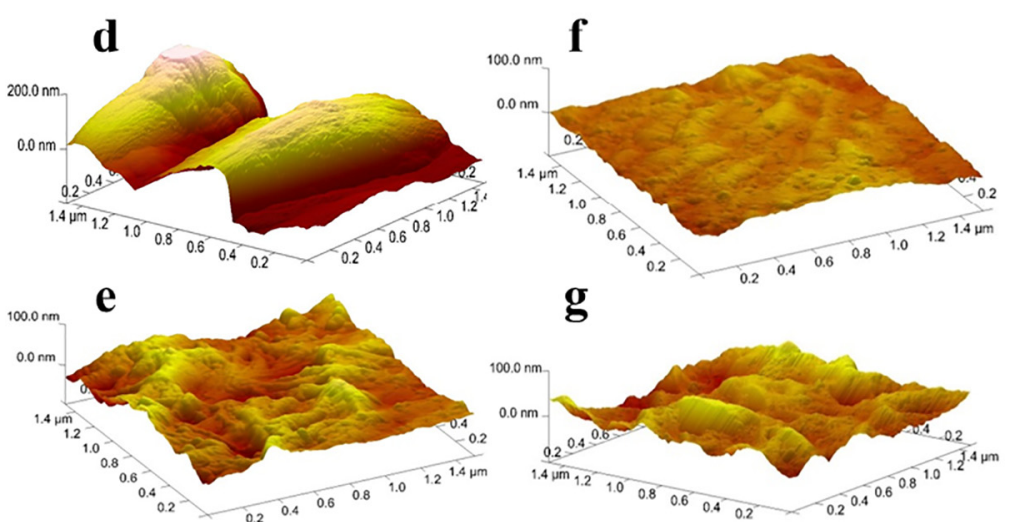

g

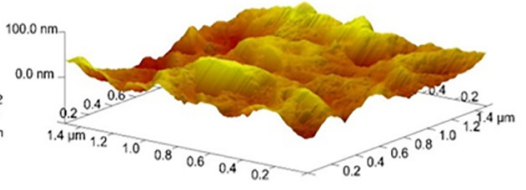

h

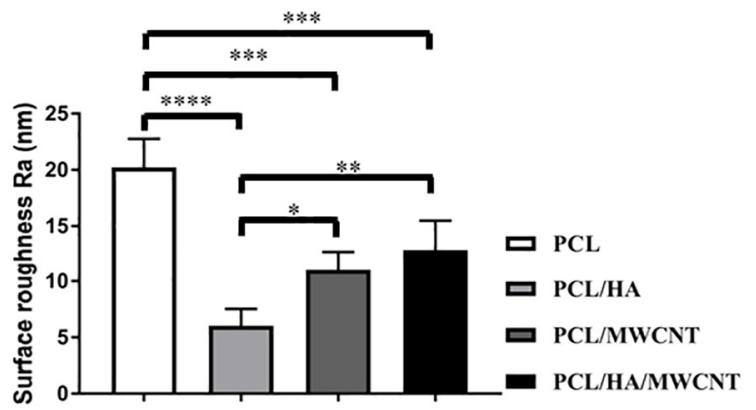

Fig. 2. SEM images showing (a) PCL/HA/MWCNTs and (b) PCL/HA scaffold architecture and filament surface; (c) EDS analysis showing calcium distribution and Ca/ $\mathrm{P}$ ratio by scanning a point and area on the PCL/HA/MWCNTs surface; AFM analysis showing surface topography of (d) PCL, (e) PCL/MWCNTs, (f) PCL/HA, (g) PCL/ HA/MWCNTs, and (h) the surface roughness (Ra) values for all scaffolds.
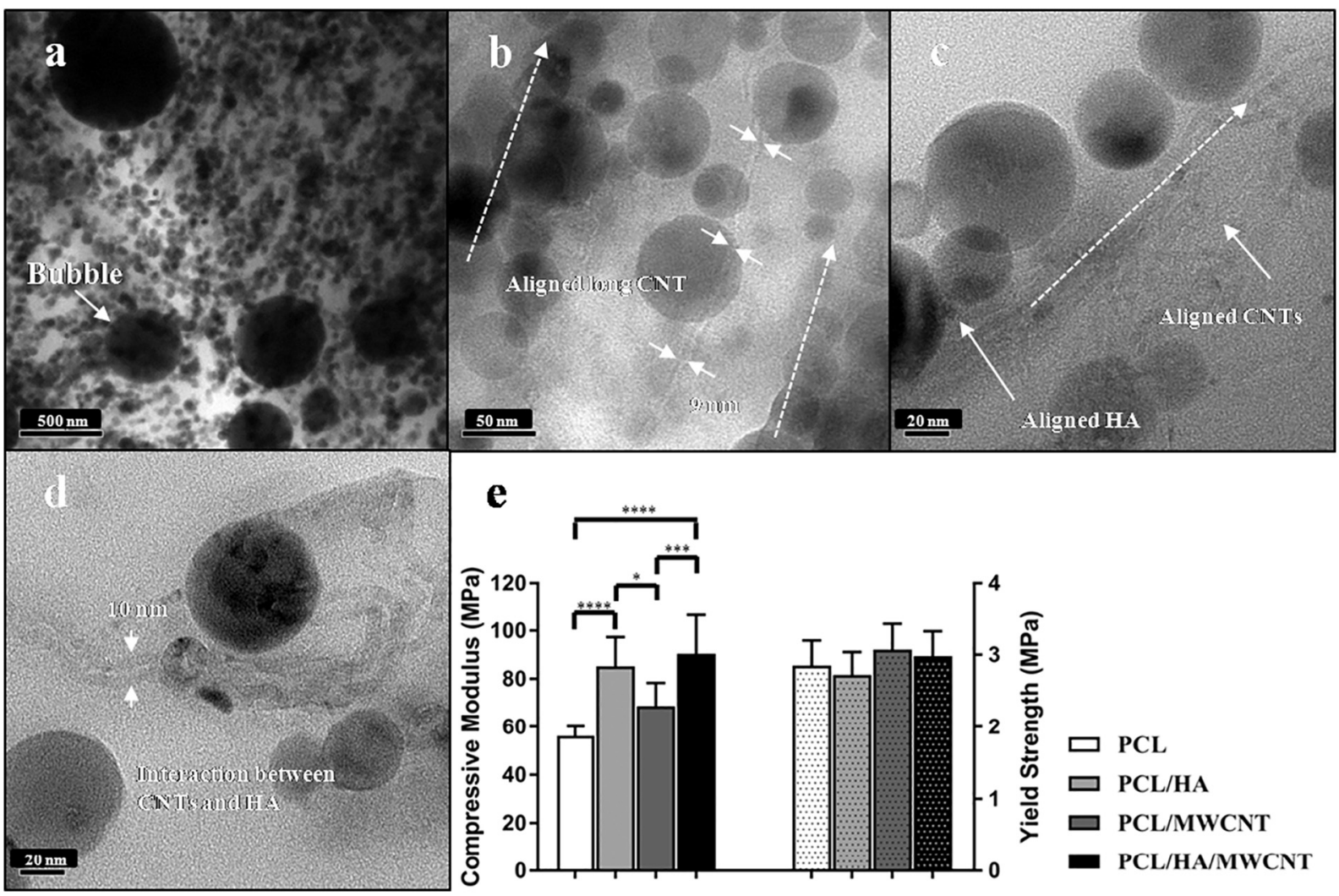

Fig. 3. (a-d) TEM analysis showing internal structure of printed filaments of PCL/HA/MWCNTs; (e) Mechanical properties of the scaffolds showing the compressive modulus and yield strength. 
to the samples and incubated for $45 \mathrm{~min}$ in the dark. The nuclear staining was performed by adding $1 \mu \mathrm{g} / \mathrm{mL}$ of DAPI and incubating for $5 \mathrm{~min}$ in the dark.

\subsection{Statistical analysis}

For all experiments, the statistical analysis was performed using one-way analysis of variance (ANOVA) with Tukey post-hoc test in GraphPad Prism software (Graphpad Software Inc., San Diego, CA, USA). Differences were considered significant at $* \mathrm{P}<0.05$, $* * \mathrm{P}<0.01, * * * \mathrm{P}<0.001$ and $* * * * \mathrm{P}<0.0001$.

\section{Results}

\subsection{Scaffold morphology and topography}

SEM images (Fig. 2a and b and Figs. S1a and S1b) show that all scaffolds were successfully produced with uniform pore shape and distribution, sharing a similar architecture at the macro-scale level. The filaments in the PCL/HA/MWCNT scaffold show a variation in shape. EDS analysis (Fig. 2c and Fig. S1d) show the presence of HA particles on both PCL/HA and PCL/HA/MWCNT surfaces with similar $\mathrm{Ca} / \mathrm{P}$ ratios. The nHA particles are homogeneously distributed on the surface of the filament. Results also suggest that the addition of MWCNTs has limited impact on the HA dispersion in the polymer matrix.

Surface topography and roughness determined by AFM are shown in Fig. 2d-h and Fig. S2. A significant surface undulation is observed for PCL scaffolds, presenting the largest surface roughness (Ra $\sim 20 \mathrm{~nm}$ ). These surface characteristics are modified by the addition of both nHA and MWCNTs with a smaller surface undulation on the PCL/HA, PCL/ MWCNTs and PCL/HA/MWCNTs scaffolds. PCL/HA scaffolds present the smallest surface roughness while multiple small embossments can be observed on the PCL/MWNCTs scaffold. The addition of both HA and MWCNTs into the PCL matrix increases the surface roughness in comparison to PCL/HA and PCL/MWCNTs, but still statistically lower than the surface roughness of PCL scaffolds.

\subsection{Transmission electron microscopy}

Fig. 3a-d shows the internal structure of PCL/HA/MWCNTs nanocomposites determined by TEM. The images show that the nHA particles are well dispersed in the polymer matrix (Fig. 3a). Some internal air bubbles can also be found in the polymer matrix generated during the printing process. The nHA is homogenously distributed in the matrix and a range of particle sizes can be observed $(<500 \mathrm{~nm})$. Large aspect ratio MWCNTs can be seen in Fig. 3b-d with diameters typically below $20 \mathrm{~nm}$. Results also show that both nHA and MWCNTs are aligned along the printing direction (Fig. $3 \mathrm{~b}$ and c).

\subsection{Mechanical analysis}

Fig. 3e shows that the addition of nHA into the PCL matrix can significantly improve the mechanical properties of the printed scaffolds. PCL/MWCNTs show a smaller compressive modulus than PCL/HA but still higher than PCL scaffolds. The addition of MWCNTs slightly increases the yield strength of the scaffold, however, no significant differences are observed between samples.

\subsection{Polarised Raman analysis}

Polarised Raman was used to evaluate the alignment of MWCNTs in both printed filaments and pre-printed composite blends. The maximum spectrum intensity can be obtained when the incident laser beam is parallel to the MWCNTs orientation while the minimum intensity occurs when the incident laser beam is perpendicular to the direction of MWCNTs. Multiple phase angles ranging from $0^{\circ}$ (aligned with the printing direction) to $90^{\circ}$ (perpendicular to the printing direction) with intervals of $22.5^{\circ}$ are considered. Results show that the maximum intensity is obtained at $0^{\circ}$ and the minimum intensity occurs at $90^{\circ}$. Moreover, there is a decreasing trend of intensity with increasing phase angle at the characteristic $\mathrm{G}^{\prime}$ band of MWCNTs, indicating that the MWCNTs tend to be aligned along the printing direction, as observed in Fig. 4a and b. The representative intensity at each phase angle presented in Table 1 also quantitatively shows a decreasing trend. As observed from Fig. 4c, most of the area in the Raman mapping are green (intensity arbitrary values ranging from 906.55 (phase angle of $22.5^{\circ}$ ) and 1136.29 (phase angle of $0^{\circ}$ )) indicating that most MWCNTs are aligned along one direction and homogeneously dispersed in the PCL matrix. The dark red zones in the Raman map, associated with high intensities (above 1136.29 arbitrary unit), might be related to the high phase angle alignment and/or aggregation of MWCNTs. Fig. 4d and Table 1 show that the intensity of the characteristic $\mathrm{G}^{\prime}$ band is mainly (99.92\%) in the region of 767.56-1136.29 arbitrary units, indicating nearly all MWCNTs are in the region of $-45^{\circ}-45^{\circ}$. Moreover, the highest frequency occurs at $22.5^{\circ}$ showing that this corresponds to the preferential orientation. Fig. 4e shows the intensities of adjacent printed layers, which are perpendicular to each other with a phase angle fixed at $0^{\circ}$. A sharp decrease of intensity occurs when the polarised laser moves from the first layer to the next layer with the same angle. However, there is no significant differences of the $\mathrm{G}^{\prime}$ band peak intensities between $0^{\circ}$ and $90^{\circ}$ phase angles for the composite blend before the printing (Fig. 4f), indicating a random dispersion of MWCNTs in the PCL matrix.

\subsection{In vitro study}

\subsubsection{Cell proliferation}

As shown in Fig. 5a, the fluorescence intensity increases with time in all scaffolds, showing that all samples are cytocompatible and able to support cell attachment and proliferation. At day 1, PCL scaffold present a significantly higher fluorescence intensity than PCL/HA and PCL/MWCNTs scaffolds while the differences between them become less apparent at day 3. At day 7, the fluorescence intensities for PCL/ HA, PCL/MWCNTs and PCL/HA/MECNTs are significantly higher than PCL scaffolds, showing more metabolic activity implying that cells are proliferating faster on these scaffolds. Among all samples, PCL/HA/ MWCNTs scaffolds present the highest fluorescence intensity values, indicating improved cell affinity compared to other scaffolds.

\subsubsection{Alkaline phosphatase}

ALP was quantified at day 1,7 and 14 and normalised to total protein concentration as shown in Fig. 5b (Fig. S3a shows the nonnormalised values which all increase with time). At day 1, PCL and PCL/MWCNTs present statistically higher normalised ALP values than PCL/HA and PCL/HA/MWCNTs. At day 7, PCL/MWCNTs scaffolds show higher normalised ALP values compared to other scaffolds, indicating that the addition of MWCNTs improves hADSCs differentiation. PCL/HA and PCL/HA/MWCNTs scaffolds show lower ALP activity than PCL scaffolds, suggesting that the addition of HA might inhibit the ALP secretion from cells. At day 14, all scaffolds present high values of ALP, showing that an increasing number of hADSCs are differentiating towards an osteogenic lineage. Furthermore, PCL/HA/MWCNTs scaffolds show higher ALP values while the PCL/HA scaffold presents lower ALP values comparing to other samples, indicating that the addition of MWCNTs has a dominant effect on the production of ALP.

\subsubsection{Collagen}

Normalised COL was quantified at mid-late stage of osteogenesis differentiation at day 14, 21 and 28 as shown in Fig. 5c. At day 14, PCL/ HA/MWCNTs present statistically higher values of COL than the other samples while PCL show statistically the lower values. PCL/HA presents slightly higher normalised COL values than PCL/MWCNTs. A similar 

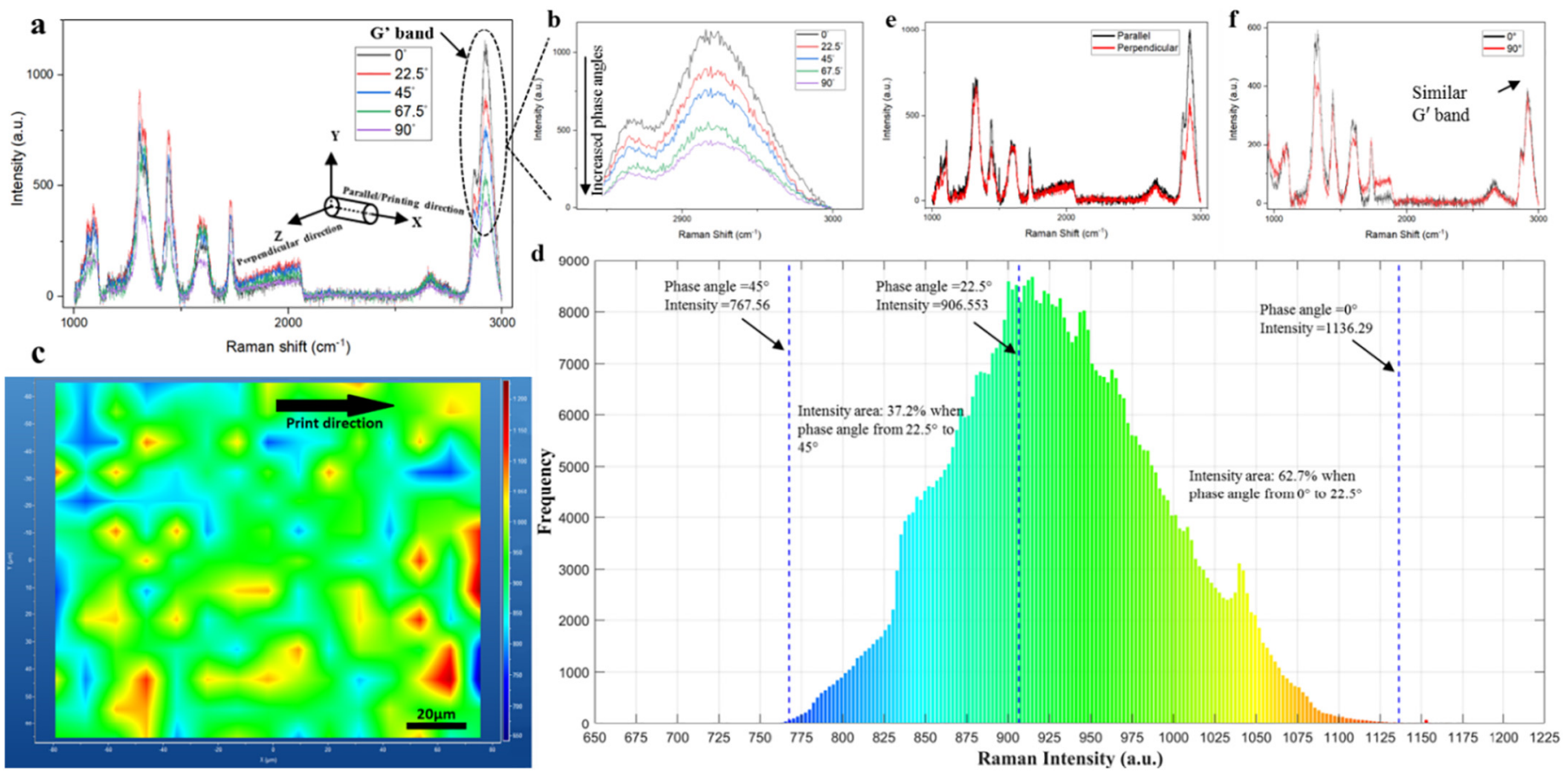

Intensity (a.u.)

Fig. 4. Polarised Raman showing (a) the decrease of the characteristic MWCNT peak of the G' band with the increase of the polarised angle; (b) Highlighted G' band peak; (c) Polarised Raman mapping showing the intensities of the $\mathrm{G}^{\prime}$ band within a $120 \mu \mathrm{m} \times 180 \mu \mathrm{m}$ surface area; (d) The histogram showing the semi-quantification of the intensity area of $\mathrm{G}^{\prime}$ band with the phase angle varying from $0^{\circ}$ to $90^{\circ}$; (e) Intensity change of adjacent layers with a $0^{\circ}$ phase angle; and (f) polarised Raman for the composite blend before printing.

Table 1

Representative intensity at each phase angle and MWCNTs orientation segments.

\begin{tabular}{llllll}
\hline Phase angle & $0^{\circ}$ & $22.5^{\circ}$ & $45^{\circ}$ & $67.5^{\circ}$ & $90^{\circ}$ \\
\hline Intensity & 1136.29 & 906.55 & 767.56 & 551.32 & 433.58 \\
$\begin{array}{c}\text { MWCNT } \\
\begin{array}{c}\text { Orientation } \\
\text { Population }\end{array}\end{array}$ & Segment A & Segment B & Segment C & Segment D & Total \\
\hline
\end{tabular}

trend is observed at day 21 and 28 but the differences between each sample seem to be smaller. Results also show that there is a decreasing trend in terms of the normalised COL from day 14 to day 28 while the COL concentrations and non-collagenous proteins concentration remain similar or slightly increase at the same period as shown in Figs. S3b and S3c. These might be attributed to fact that the increased number of cells (indicated by the increased non-collagenous protein) lead to less interactions between cells and the scaffold surface.

\subsubsection{Osteocalcin}

OCN production is show in Fig. 5c and at day $1 \mathrm{PCL} / \mathrm{HA} / \mathrm{MWCNTs}$ present statistically higher values of OCN while PCL and PCL/MWCNTs show the lowest values. Similar trend is observed at day 7. At day 14, PCL/HA scaffold shares similar value with PCL/HA/MWCNT but statistically higher than PCL and PCL/MWCNTs scaffolds. At day 28, PCL/ HA/MWCNTs and PCL/HA shows statistically higher values than PCL sample. The non-normalised OCN increases in all samples with the time, indicating that hADSCs are differentiating into osteoblasts (Fig. S3d). The results seem to indicate that the addition of HA promotes OCN secretion from the differentiating hADSCs and the combination of both MWCNTs and HA can further enhance it.

\subsubsection{Mineralisation}

At day 1, relatively high calcification is observed in both PCL/HA and PCL/HA/MWCNTs scaffolds as shown in Fig. 5d. However, by analysing the non-seeded (Fig. 5e) and seeded scaffolds (Fig. 5f), it is possible to observe that the scaffold mineralisation process is mainly attributed to the presence of HA. The contribution from the cells at day 1 is limited (Fig. 5f). At day 7, results indicate that the differentiated hADSCs are increasingly producing calcium and thus contributing to the mineralisation of the scaffolds. The overall ARS values of PCL/HA and PCL/HA/MWCNTs scaffolds are statistically higher than PCL and PCL/MWNCTs scaffolds. However, when subtracting the ARS values of the non-seeded scaffolds from the overall ARS values, it is possible to observe that the mineralisation activities of differentiated hADSCs are significantly higher in the case of PCL and PCL/MWCNTs scaffolds. PCL/MWCNTs scaffolds present a higher value than PCL scaffolds. This trend is also observed at day 14 with PCL and PCL/MWCNTs scaffolds showing higher ARS values in comparison to PCL/HA and PCL/HA/ MWCNTs scaffolds, after removing the scaffold calcium content and self-mineralisation effect. These results seem to indicate that MWCNTs stimulates the differentiated hADSCs to induce mineralisation of the scaffold while the addition of nHA has limited impact on the cellular induced mineralisation of the scaffold.

SEM images for PCL/MWCNTs and PCL/HA/MWCNTs scaffolds after 21 days of cell differentiation are shown in Fig. 6a-f. EDS linear scanning (Fig. 6d) shows the scaffold surface composition after cell seeding and differentiation. nHA containing scaffolds present a layer of intensive and compact calcium nodules on the surface. However, in contrast this isn't observed as clearly on the PCL/MWCNTs scaffold. Although calcium nodules are also dispersed on the surfaces of the PCL/ MWCNTs scaffolds, these nodules are mainly distributed around the cell membrane or entrapped by the cell membrane. It is important to note that these calcium nodules are generated not only by the cells during the differentiation process but also by the scaffold self-mineralisation process due to the incubation in culture media and then osteogenic media (Fig. $6 \mathrm{~g}$ and $\mathrm{h}$ ). These calcium nodules also act as preferential local cell adhesion points (Fig. 6f). EDS analysis further confirms the different surface characteristics as shown in Fig. 6b and e. It can be observed that both calcium and phosphate elements increase in the areas where the cell membrane is scanned in the PCL/MWCNTs scaffolds (Fig. 6b). However, Fig. 6e shows a sudden increase of calcium 

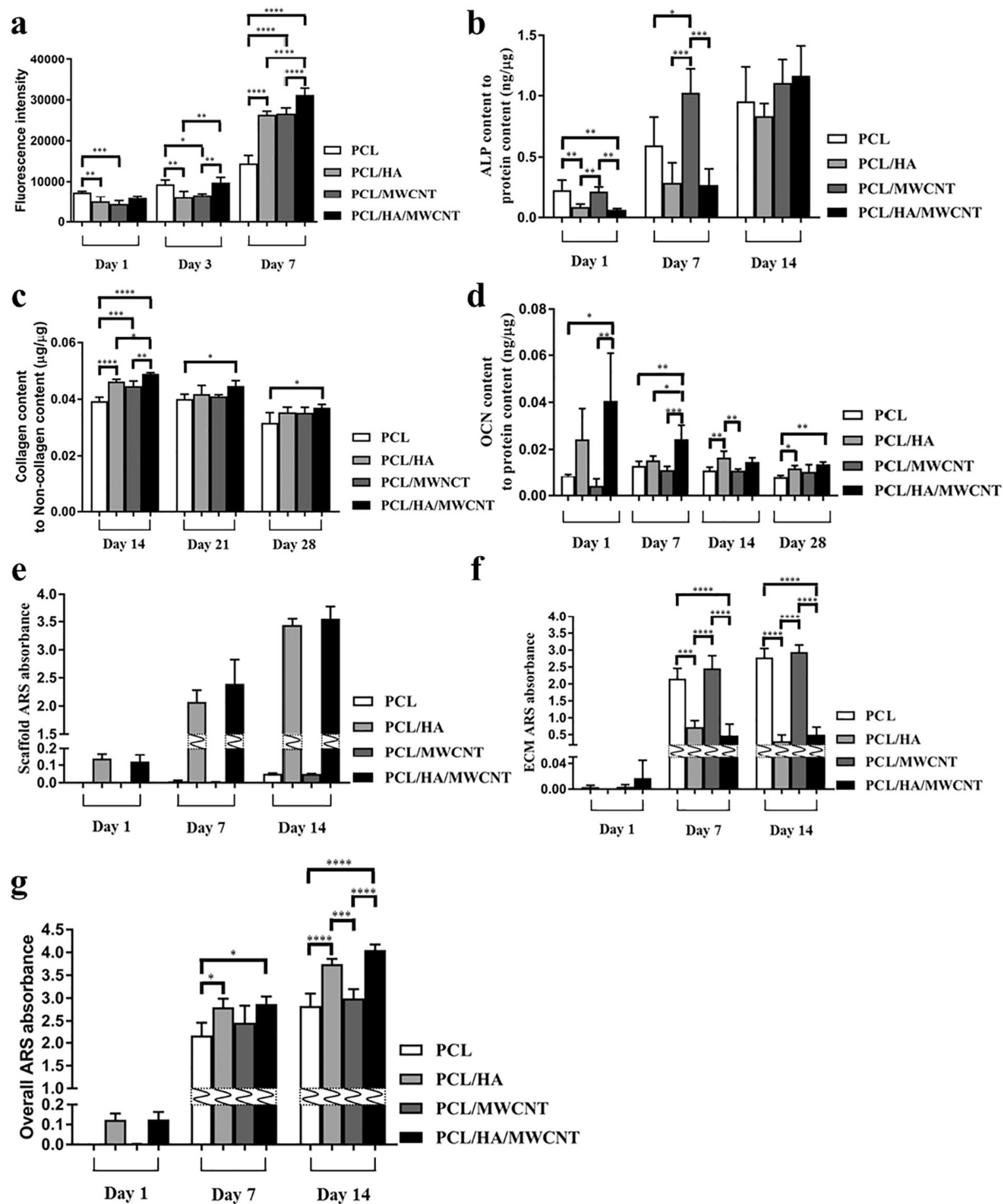

Fig. 5. (a) Alamar Blue assay showing the fluorescence intensity of all scaffolds at day 1,3 and 7; (b) ALP production normalised to total protein for all samples at day 1, 7 and 14; (c) Total collagen content normalised to non-collagen protein content for all samples at day 14, 21 and 28 ; (d) OCN content normalised to total protein for all samples at day 1, 7, 14 and 28; (e) ARS staining showing inherent calcium content and self-mineralisation of non-cell seeded scaffolds at day 1, 7 and 14; ( $\mathrm{g}$ ) ARS staining showing calcium formation produced by cells for all samples at day 1, 7 and 14; (e) Total ARS staining for all scaffolds at day 1,7 and 14 .

and phosphate and a decrease of carbon in the absence of cells, indicating high level of self-mineralisation and the inherent mineral content on the surface of the scaffolds containing nHA particles. Confocal images of the PCL/HA/MWCNTs scaffold (Fig. 6i) shows cells that have a spread morphology as observed by the stained actin filaments (green) which are extended and elongated on the filament surface and the nuclei staining (blue) indicates that the cells have migrated and proliferated throughout the scaffold. Cell bridging and cell migration from the top to the next layer can also be observed, indicating that the scaffold is able to support cell attachment, growth, and migration.

\section{Discussion}

Additive manufacturing or $3 \mathrm{D}$ printing is a highly relevant 

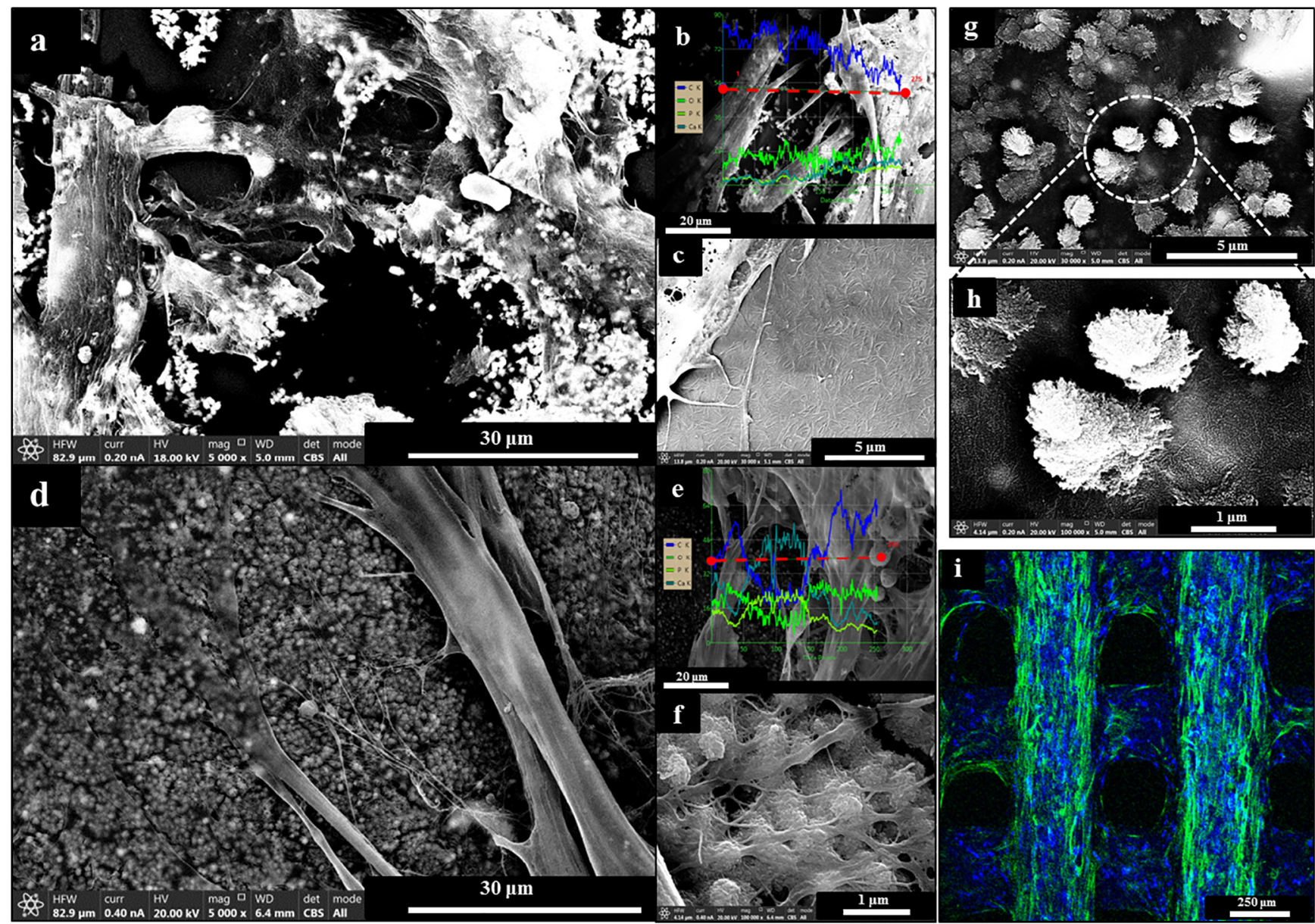

Fig. 6. Cell-scaffold interactions after 21 days differentiation. (a) SEM image showing cells on PCL/MWCNTs; (b) EDS linear analysis showing the surface composition of PCL/MWCNTs scaffold; (c) High magnification SEM images of PCL/MWNCTs scaffold; (d) SEM image showing cell-scaffold interaction on PCL/HA/ MWCNTs scaffold; (e) EDS linear analysis showing the surface composition of PCL/HA/MWCNTs scaffold; (f) High magnification SEM images of PCL/HA/MWNCTs scaffold; (g-h) SEM image showing self-mineralisation on the surface of scaffolds containing nHA; (i) Confocal image showing cell distribution on the PCL/HA/ MWCNTs scaffold. (Red line: EDS scanning routine). (For interpretation of the references to colour in this figure legend, the reader is referred to the Web version of this article.)

manufacturing technology for tissue engineering applications due to the ability to produce complex, multi-material, and hierarchical 3D scaffolds. In this study, an extrusion-based additive manufacturing system successfully produced hierarchical scaffolds with uniform geometry at a micro scale and highly aligned structures at the nano-scale, mimicking the bone structure from macro to nano-scale (Fig. 7a and b). The MWCNTs are mostly aligned along the printing direction and surrounded by nano-scale synthetic HAs, establishing a collagen fibril-like structure, with the aligned long MWCNTs analogous to the collagen fibril and the synthetic nHA acting as the natural bone HA crystals. TEM and polarised Raman analysis confirm that MWCNTs are highly aligned along the printing direction (Fig. 3b and Fig. 4) with nHA well dispersed around the MWCNTs (Fig. 3c).

In comparison to both PCL and PCL/HA scaffold, PCL/MWCNTs and PCL/HA/MWCNTs scaffolds demonstrate inconsistency in the filament shape related to the rheological properties of the nanocomposite melt, as previously reported [24]. This leads to surface distortions during the extrusion process. The addition of MWCNTs to a PCL matrix significantly increases the storage modulus, which represents a solid-like behaviour, thus the composite material becomes stiffer and the flow properties change.

As previously reported, nHA particles (below $200 \mathrm{~nm}$ ) tend to form agglomerations, in the powder form, with an average particle size of approximately $3.41 \mu \mathrm{m}$ due to strong Van der Waals forces [25]. These agglomerations seem to be eliminated during the printing process due to the mixing by the screw (Fig. 2c). This is confirmed by TEM, showing an overall homogeneous distribution of nHA in the polymer matrix (Fig. 3a). AFM results show that the addition of MWCNTs significantly increases the surface roughness compared to the addition of nHA only. Variations in crystallinity lead to changes in surface roughness and, as previously reported, the polymer chains in the CNTs' vicinity (interphase) are more packed and present a higher orientation than the bulk polymer $[26,27]$. The high aspect ratio of MWCNTs further strengthens this behaviour. Therefore, it can be assumed that MWCNT bundles act as nucleating sites allowing the recrystallisation of PCL chains along the wall of the nanotubes, thus increasing the surface roughness. The addition of nHA into the PCL/MWCNT composition further increases the crystal size. Although HA is also considered to be a nucleating agent for polymer recrystallisation [28,29], the MWCNTs effect seems to be dominant. Mechanical analysis shows that the addition of HA has a significant impact in comparison to the MWCNTs due to the low concentration of MWCNTs. In the printed scaffolds, synthetic nHA plays a similar role as the HA crystals in the natural bone by providing improved mechanical properties. We have previously shown that the inclusion of MWCNTs increases the nanoscale hardness and elastic modulus of the filament [24]. This may contribute to the promotion of osteogenic differentiation due to the stiffer surface modulating mechanotransduction pathways [30,31].

Cells are sensitive to the surface topography, which has a significant impact on cellular activities including cell morphology, migration, 


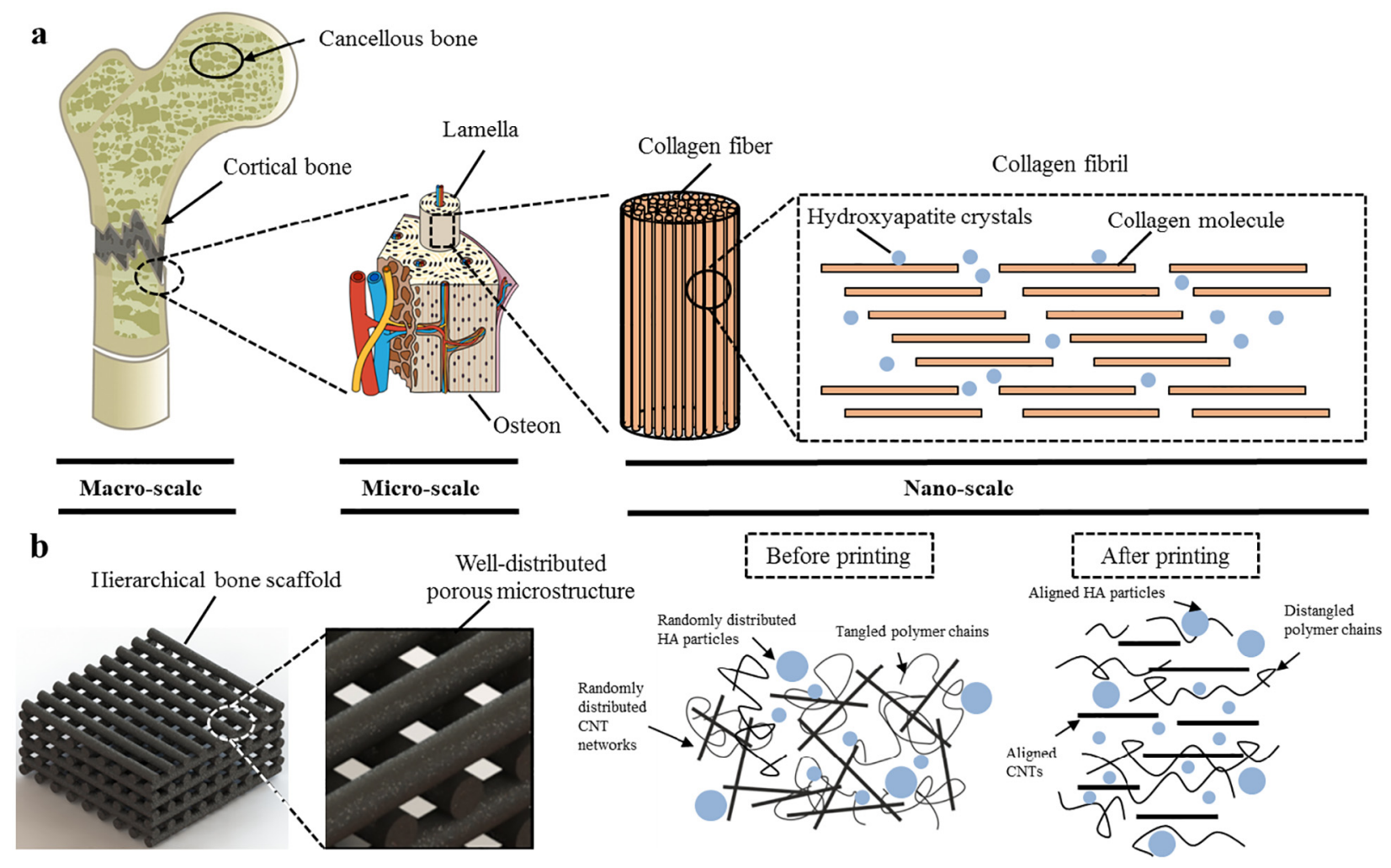

Fig. 7. Schematic representation showing (a) the hierarchical bone structure from macro-size to nano-size (b) which is mimicked by the biomimetic 3D printed PCL/ HA/MWCNT hierarchical scaffolds.

proliferation and differentiation [32]. However, there are contradictory results regarding how surface roughness influences cell proliferation. Deligianni et al. [33] investigated cell proliferation on HA substrates using human bone marrow cells and showed that cell proliferation increases with the increase in the surface roughness. Contrary, Washburn et al. [34] reported high osteoblastic MC3T3-E1 cell proliferation in smooth poly(L-lactic acid) substrates. In this study, results seem to indicate that cell proliferation increases with increasing surface roughness. However, the low cell proliferation rate observed in the rough PCL scaffolds indicates that cell proliferation is also material dependent. Previously, we reported that the addition of MWCNTs into the PCL matrix promotes protein adsorption possibly due to the non-covalent $\pi$ $\pi$ stacking interactions between the graphitic structure and the aromatic rings of proteins and also provides a stiffer surface, modulating mechanotransduction pathways, which allowed for increased cell proliferation [24]. This may explain the higher cell proliferation observed on PCL/MWCNTs and PCL/HA/MWCNTs scaffolds.

Osteogenic cells arise from multipotent mesenchymal stem cells and follow the differentiation sequence from osteoprogenitor, pre-osteoblast, osteoblast and osteocyte [35-37]. Pre-osteoblast is considered to be highly positive for ALP activity and the transition from pre-osteoblast to mature osteoblast is characterised by a high expression of bone matrix protein such as OCN [38]. Results show that the ALP activity increases from day 1 to day 14 while the OCN secretions are relatively low, indicating that the differentiated cells are at a pre-osteoblast stage (Fig. 5 and Fig. S3d). The ALP activities for non-HA scaffolds are significantly higher than the ALP activities for scaffolds containing nHA, particularly at early stage of cell differentiation (day 1 and day 7). A similar trend is also observed in Fig. 5f, showing a significantly higher bone matrix calcification in non-HA scaffolds. This trend might be attributed to the fact that the calcium in the bone matrix of non-HA scaffolds can easily acquire phosphates arisen from the increase of ALP activity and the increased degradation of $\beta$-glycerophosphate [39]. Optimisation of calcium concentration is crucial in the design of bone scaffolds as high calcium concentrations have been reported to inhibit early-stage markers of osteogenic differentiation (ALP), however, latestage markers (OCN) were promoted instead [40-42]. In this study, the osteogenic media provides calcium and phosphate while the addition of nHA offers an extra calcium and phosphate source due to its intrinsic chemical composition as well as scaffold self-calcification, which creates a localised high calcium environment for hADSCs differentiation as shown in Fig. 6e. This may explain the reduced ALP activity in scaffolds containing nHA in early stage of osteogenic differentiation (day 1 and day 7). However, from Fig. 5b it is possible to observe that at day 14, PCL/HA/MWNCTs scaffolds exhibit slightly higher ALP activity comparing to the other scaffolds. Moreover, scaffolds containing nHA present similar COL and OCN values comparing to PCL/MWCNTs scaffold and higher values than PCL scaffolds. These results seem to indicate that high calcium concentration might increase markers of mid-late stage of osteogenic differentiation. The self-calcification of scaffolds containing nHA also influences the ARS results. The mineralisation effect of nHA is shown in Fig. $5 \mathrm{f}$ and $\mathrm{g}$. Results show that nHA promotes the overall scaffold calcification but seems to inhibit the cellular induced mineralisation process. However, this also might attribute to the limitation of measurement technique as the ARS staining solution is not able to penetrate the thick cell layer and stain the self-mineralised scaffolds. In addition, cell differentiation is also sensitive to surface topography, reported to be proportional to the surface roughness $[29,34,43,44]$ and also dependent on the geometry of the nano-topographic substrate [32]. Therefore, the nanograting-like surface structures created by the long MWCNTs seem to influence cellular behaviour (Fig. 2e and g). This can be confirmed by the confocal images presented in Fig. 6i, showing elongated cells parallel to the printing direction, which is a typical response of different cell types to nano-gratings [32]. Moreover, these nano-grating structures may act as physical cues influencing cell mechanotransduction and inducing cell differentiation through focal adhesion kinase signalling pathway [45]. Osteogenic differentiation is a multi-component pathway derived from physical 
and chemical cues. The hierarchical nanocomposite PCL/HA/MWCNT scaffold provides a variety of topographical, mechanical, and chemical cues stimulating osteogenic differentiation, however, further studies are required to understand these signalling pathways.

\section{Conclusion}

PCL and PCL composite scaffolds containing HA, MWCNTs and HA/ MWCNTs were successfully produced using screw-assisted extrusionbased additive manufacturing. As observed by TEM and polarised Raman, the printed PCL/HA/MWCNTs scaffold presents an analogue nanostructure to natural bone. Results also show the addition of HA and MWCNTs improve both mechanical properties and cellular activities including cell proliferation, differentiation and mineralisation. Moreover, the addition of HA particles allows an overall improvement of the scaffolds mineralisation but lowers the early stage of cell differentiation. The addition of MWCNTs shows a dominant effect on osteogenic differentiation and promotes ALP, COL and OCN expressions in PCL/HA/MWCNTs scaffolds. Despite the positive results reported in this paper further research is still required to optimise the concentration of both MWCNTs and HA targeting to obtain an optimal ratio not only to enhance physiochemical properties but also to promote the cellular response.

\section{Declaration of competing interest}

There is no conflict of Interest.

\section{Acknowledgement}

This project was partially supported by the University of Manchester/King Saud University research grant "Multi scale bioactive scaffolds for bone regeneration" and the Engineering and Physical Sciences Research Council and the Medical Research Council Centre for Doctoral Training in Regenerative Medicine (EP/L014904/1).

\section{Appendix A. Supplementary data}

Supplementary data to this article can be found online at https:// doi.org/10.1016/j.msec.2019.110374.

\section{References}

[1] B.S. Harrison, A. Atala, Carbon nanotube applications for tissue engineering, Biomaterials 28 (2) (2007) 344-353.

[2] F.P. Melchels, M.A. Domingos, T.J. Klein, J. Malda, P.J. Bartolo, D.W. Hutmacher, Additive manufacturing of tissues and organs, Prog. Polym. Sci. 37 (8) (2012) 1079-1104.

[3] P. Bartolo, J.-P. Kruth, J. Silva, G. Levy, A. Malshe, K. Rajurkar, et al., Biomedical production of implants by additive electro-chemical and physical processes, CIRP Ann. - Manuf. Technol. 61 (2) (2012) 635-655.

[4] H. Naderi, M.M. Matin, A.R. Bahrami, Critical issues in tissue engineering: biomaterials, cell sources, angiogenesis, and drug delivery systems, J. Biomater. Appl. 26 (4) (2011) 383-417.

[5] P. Newman, A. Minett, R. Ellis-Behnke, H. Zreiqat, Carbon nanotubes: their potential and pitfalls for bone tissue regeneration and engineering, Nanomed. Nanotechnol. Biol. Med. 9 (8) (2013) 1139-1158.

[6] N. Reznikov, R. Shahar, S. Weiner, Bone hierarchical structure in three dimensions, Acta Biomater. 10 (9) (2014) 3815-3826.

[7] M. Kim, Y. Choe, G. Kim, Injectable hierarchical micro/nanofibrous collagen-based scaffolds, Chem. Eng. J. 365 (2019) 220-230.

[8] T. Bian, K. Zhao, Q. Meng, Y. Tang, H. Jiao, J. Luo, The construction and performance of multi-level hierarchical hydroxyapatite (HA)/collagen composite implant based on biomimetic bone Haversian motif, Mater. Des. 162 (2019) 60-69.

[9] Y. Zhao, J. Chen, L. Zou, G. Xu, Y. Geng, Facile one-step bioinspired mineralization by chitosan functionalized with graphene oxide to activate bone endogenous regeneration, Chem. Eng. J. 378 (2019) 122174.

[10] S. Hou, X. Niu, L. Li, J. Zhou, Z. Qian, D. Yao, et al., Simultaneous nano- and microscale structural control of injectable hydrogels via the assembly of nanofibrous protein microparticles for tissue regeneration, Biomaterials 223 (2019) 119458.

[11] S. Samavedi, A.R. Whittington, A.S. Goldstein, Calcium phosphate ceramics in bone tissue engineering: a review of properties and their influence on cell behavior, Acta
Biomater. 9 (9) (2013) 8037-8045

12] D. Toroian, J.E. Lim, P.A. Price, The size exclusion characteristics of type I collagen implications for the role of noncollagenous bone constituents in mineralization, J. Biol. Chem. 282 (31) (2007) 22437-22447.

[13] M.E. Launey, M.J. Buehler, R.O. Ritchie, On the mechanistic origins of toughness in bone, Annu. Rev. Mater. Res. 40 (2010) 25-53.

[14] S. Liao, G. Xu, W. Wang, F. Watari, F. Cui, S. Ramakrishna, et al., Self-assembly of nano-hydroxyapatite on multi-walled carbon nanotubes, Acta Biomater. 3 (5) (2007) 669-675.

[15] A. Oyefusi, O. Olanipekun, G.M. Neelgund, D. Peterson, J.M. Stone, E. Williams, et al., Hydroxyapatite grafted carbon nanotubes and graphene nanosheets: promising bone implant materials, Spectrochim. Acta A Mol. Biomol. Spectrosc. 132 (2014) 410-416.

[16] I.-K. Yoon, J.-Y. Hwang, J-w Seo, W.-C. Jang, H.-W. Kim, U.S. Shin, Carbon nanotube-gelatin-hydroxyapatite nanohybrids with multilayer core-shell structure for mimicking natural bone, Carbon 77 (2014) 379-389.

[17] L. Meng, C. Fu, Q. Lu, Advanced technology for functionalization of carbon nanotubes, Prog. Nat. Sci. 19 (7) (2009) 801-810.

[18] E.M. Gonçalves, F.J. Oliveira, R.F. Silva, M.A. Neto, M.H. Fernandes, M. Amaral, et al., Three-dimensional printed PCL-hydroxyapatite scaffolds filled with CNTs for bone cell growth stimulation, J. Biomed. Mater. Res. B Appl. Biomater. 104 (6) (2016) 1210-1219.

[19] Z. Jing, Y. Wu, W. Su, M. Tian, W. Jiang, L. Cao, et al., Carbon nanotube reinforced collagen/hydroxyapatite scaffolds improve bone tissue formation in vitro and in vivo, Ann. Biomed. Eng. 45 (9) (2017) 2075-2087.

[20] K. Zhou, P. Yu, X. Shi, T. Ling, W. Zeng, A. Chen, et al., Hierarchically porous hydroxyapatite hybrid scaffold incorporated with reduced graphene oxide for rapid bone ingrowth and repair, ACS Nano 13 (8) (2019) 9595-9606.

[21] W.-H. Chiang, D.N. Futaba, M. Yumura, K. Hata, Growth control of single-walled, double-walled, and triple-walled carbon nanotube forests by a priori electrical resistance measurement of catalyst films, Carbon 49 (13) (2011) 4368-4375.

[22] G. Heo, Y.S. Kim, S.-H. Chun, M.-J. Seong, Polarized Raman spectroscopy with differing angles of laser incidence on single-layer graphene, Nanoscale Res. Lett. 10 (1) (2015) 45

[23] N.R. Raravikar, L.S. Schadler, A. Vijayaraghavan, Y. Zhao, B. Wei, P.M. Ajayan, Synthesis and characterization of thickness-aligned carbon nanotube - polymer composite films, Chem. Mater. 17 (5) (2005) 974-983.

[24] B. Huang, C. Vyas, I. Roberts, Q.-A. Poutrel, W.-H. Chiang, J.J. Blaker, et al., Fabrication and characterisation of 3D printed MWCNT composite porous scaffolds for bone regeneration, Mater. Sci. Eng. C 98 (2019) 266-278.

[25] B. Huang, P.J. Bártolo, Rheological characterization of polymer/ceramic blends for 3D printing of bone scaffolds, Polym. Test. 68 (2018) 365-378.

[26] J. Meng, Y. Zhang, K. Song, M.L. Minus, Forming crystalline polymer-nano interphase structures for high-Modulus and high-Tensile/Strength composite fibers, Macromol. Mater. Eng. 299 (2) (2014) 144-153.

[27] Y. Liu, S. Kumar, Polymer/carbon nanotube nano composite fibers-a review, ACS Appl. Mater. Interfaces 6 (9) (2014) 6069-6087.

[28] S. Mollazadeh, J. Javadpour, A. Khavandi, In situ synthesis and characterization of nano-size hydroxyapatite in poly (vinyl alcohol) matrix, Ceram. Int. 33 (8) (2007) 1579-1583.

[29] A. Patlolla, G. Collins, T.L. Arinzeh, Solvent-dependent properties of electrospun fibrous composites for bone tissue regeneration, Acta Biomater. 6 (1) (2010) 90-101.

[30] A.J. Engler, S. Sen, H.L. Sweeney, D.E. Discher, Matrix elasticity directs stem cell lineage specification, Cell 126 (4) (2006) 677-689.

[31] Y.R. Shih, K.F. Tseng, H.Y. Lai, C.H. Lin, O.K. Lee, Matrix stiffness regulation of integrin-mediated mechanotransduction during osteogenic differentiation of human mesenchymal stem cells, J. Bone Miner. Res. : Off. J. Ame. Soc. Bone Miner. Res. 26 (4) (2011) 730-738.

[32] C.J. Bettinger, R. Langer, J.T. Borenstein, Engineering substrate topography at the micro-and nanoscale to control cell function, Angew. Chem. Int. Ed. 48 (30) (2009) 5406-5415.

[33] D.D. Deligianni, N.D. Katsala, P.G. Koutsoukos, Y.F. Missirlis, Effect of surface roughness of hydroxyapatite on human bone marrow cell adhesion, proliferation, differentiation and detachment strength, Biomaterials 22 (1) (2000) 87-96.

[34] N.R. Washburn, K.M. Yamada, C.G. Simon Jr., S.B. Kennedy, E.J. Amis, Highthroughput investigation of osteoblast response to polymer crystallinity: influence of nanometer-scale roughness on proliferation, Biomaterials 25 (7-8) (2004) 1215-1224.

[35] B.G. Demczyk, Y.M. Wang, J. Cumings, M. Hetman, W. Han, A. Zettl, et al., Direct mechanical measurement of the tensile strength and elastic modulus of multiwalled carbon nanotubes, Mater. Sci. Eng. A 334 (1) (2002) 173-178.

[36] V. Kartsogiannis, K.W. Ng, Cell lines and primary cell cultures in the study of bone cell biology, Mol. Cell. Endocrinol. 228 (1) (2004) 79-102.

[37] R. Florencio-Silva, GRdS. Sasso, E. Sasso-Cerri, M.J. Simões, P.S. Cerri, Biology of bone tissue: structure, function, and factors that influence bone cells, BioMed Res. Int. 2015 (2015).

[38] M. Capulli, R. Paone, N. Rucci, Osteoblast and osteocyte: games without frontiers, Arch. Biochem. Biophys. 561 (2014) 3-12.

[39] M. Shimizu, Y. Kobayashi, T. Mizoguchi, H. Nakamura, I. Kawahara, N. Narita, et al., Carbon nanotubes induce bone calcification by bidirectional interaction with osteoblasts, Adv. Mater. 24 (16) (2012) 2176-2185.

[40] S. Cheng, W. Wang, Z. Lin, P. Zhou, X. Zhang, W. Zhang, et al., Effects of extracellular calcium on viability and osteogenic differentiation of bone marrow stromal cells in vitro, Hum. Cell 26 (3) (2013) 114-120.

[41] S. An, J. Ling, Y. Gao, Y. Xiao, Effects of varied ionic calcium and phosphate on the 
proliferation, osteogenic differentiation and mineralization of human periodontal ligament cells in vitro, J. Periodontal. Res. 47 (3) (2012) 374-382.

[42] H. Gu, F. Guo, X. Zhou, L. Gong, Y. Zhang, W. Zhai, et al., The stimulation of osteogenic differentiation of human adipose-derived stem cells by ionic products from akermanite dissolution via activation of the ERK pathway, Biomaterials 32 (29) (2011) 7023-7033.

[43] K. Anselme, P. Linez, M. Bigerelle, D. Le Maguer, A. Le Maguer, P. Hardouin, et al, The relative influence of the topography and chemistry of TiAl6V4 surfaces on osteoblastic cell behaviour, Biomaterials 21 (15) (2000) 1567-1577.

[44] H. Chen, X. Huang, M. Zhang, F. Damanik, M.B. Baker, A. Leferink, et al., Tailoring surface nanoroughness of electrospun scaffolds for skeletal tissue engineering, Acta Biomater. 59 (2017) 82-93.

[45] B.K.K. Teo, S.T. Wong, C.K. Lim, T.Y. Kung, C.H. Yap, Y. Ramagopal, et al., Nanotopography modulates mechanotransduction of stem cells and induces differentiation through focal adhesion kinase, ACS Nano 7 (6) (2013) 4785-4798. 